\title{
Photocyclization of Iminyl Radicals: Theoretical Study and Photochemical Aspects
}

\author{
Rafael Alonso, Pedro J. Campos, Miguel A. Rodríguez, * Diego Sampedro* \\ Departamento de Química, Universidad de La Rioja, Grupo de Síntesis Química de La Rioja, \\ Unidad Asociada al C.S.I.C., Madre de Dios, 51, 26006 Logroño, Spain
}

Email: miguelangel.rodriguez@unirioja.es

\section{SUPPORTING INFORMATION \\ TABLE OF CONTENTS}
A. General Methods and Characterization Data for Compounds 2 and 11 (page 2).
B. NMR Spectra for Compounds 2, 11 and 12 (page 3).
C. Experimental Procedure for Irradiation of $\mathbf{1 1}$ in the Presence of Different Triplet Quenchers and Sensitizers (page 7).
D. Experimental Procedure for Laser Flash Photolysis Measurements (page 8).
E. Experimental Procedure for Stern-Volmer Analysis (page 9).
F. Experimental Procedure for Quantum Yield Determination (page 10).
G. Experimental Procedure for Luminescence Measurements (page 11).
H. Cartesian Coordinates for the Calculated Geometries (page 12).

\footnotetext{
* Corresponding authors. Tel.: +34-941299647; fax: +34-299621
} 
A. General Methods and Characterization Data for Compounds $\mathbf{2}$ and $\mathbf{1 1 .}$

\section{General Methods}

All solvents were purified by standard procedures. Reagents were of commercial grades. $O$-acetyloximes were prepared from ketones by the general procedure. ${ }^{1} Z-1$ phenyl-4-hepten-1-one was prepared from Z-4-heptenal by addition of phenyl magnesium bromide followed by oxidation. ${ }^{2}$

5-Hexen-2-one $\boldsymbol{O}$-acetyloxime (2). Colourless oil (obtained as a 73:27 isomer mixture). Yield: $1076 \mathrm{mg}, 90 \% .{ }^{1} \mathrm{H}$ NMR $\left(300 \mathrm{MHz}, \mathrm{CDCl}_{3}\right) \oint 5.77-5.82(\mathrm{~m}, 1 \mathrm{H}), 5.00-$ $5.10(\mathrm{~m}, 2 \mathrm{H}), 2.43-2.52(\mathrm{~m}, 2 \mathrm{H}), 2.30-2.37(\mathrm{~m}, 2 \mathrm{H}) 2.17(\mathrm{~s}, 3 \mathrm{H}), 2.17(\mathrm{~s}, 3 \mathrm{H}), 2.03(\mathrm{~s}$, $3 \mathrm{H}), 1.98(\mathrm{~s}, 3 \mathrm{H}) \mathrm{ppm} .{ }^{13} \mathrm{C} \mathrm{NMR}\left(300 \mathrm{MHz}, \mathrm{CDCl}_{3}\right) \delta 168.9,168.7,166.4,165.8$, 136.6, 136.5, 116.0, 115.9, 35.1, 30.4, 30.0, 29.8, 20.3, 19.8, 19.7, 15.5 ppm. GC-MS: $m / z: 155$. ES (+): $m / z: 156(\mathrm{M}+1)$. UV: $\lambda 202,232 \mathrm{~nm}\left(\varepsilon=12183,2218 \mathrm{M}^{-1} \mathrm{~cm}^{-1}\right)$. Anal. Calcd for $\mathrm{C}_{8} \mathrm{H}_{13} \mathrm{NO}_{2}$ : C, 61.91; H, 8.44; N, 9.03; O, 20.62. Found: C, 61.96; H, 8.39; N, 8.99; O, 20.66.

(Z)-1-Phenyl-4-hepten-1-one $\boldsymbol{O}$-acetyloxime (11). Colourless oil. Yield: 3528 $\mathrm{mg}, 72 \% .{ }^{1} \mathrm{H}$ NMR $\left(300 \mathrm{MHz}, \mathrm{CDCl}_{3}\right) \delta .72(\mathrm{~d}, J=6.0 \mathrm{~Hz}, 2 \mathrm{H}), 7.45-7.38(\mathrm{~m}, 2 \mathrm{H})$, 2.92-2.86 (m, 2H), 2.35-2.30 (m, 2H), $2.27(\mathrm{~s}, 3 \mathrm{H}), 2.02-1.96(\mathrm{~m}, 2 \mathrm{H}), 0.93(\mathrm{t}, J=$ $6.0 \mathrm{~Hz}, 3 \mathrm{H}) \mathrm{ppm} .{ }^{13} \mathrm{C} \mathrm{NMR}\left(300 \mathrm{MHz}, \mathrm{CDCl}_{3}\right): \delta 169.02,165.90,134.08,133.48$, 130.62, 128.74, 127.39, 126.68, 28.45, 24.47, 20.56, 19.96, 14.35 ppm. ES (+): m/z: 246 $(\mathrm{M}+1) . \mathrm{UV}: \lambda$ 198, 244nm $\left(\varepsilon=9236,26.129 \mathrm{M}^{-1} \mathrm{~cm}^{-1}\right)$. Anal. Calcd for $\mathrm{C}_{15} \mathrm{H}_{19} \mathrm{NO}_{2}: \mathrm{C}$, 73.44; H, 7.81; N, 5.71; O, 13.04. Found: C, 73.39; H, 7.88; N, 5.74; O, 12.99 .

\footnotetext{
${ }^{1}$ Alonso, R.; Campos, P.J.; García, B.; Rodríguez, M.A. Org. Lett. 2006, 8, 3521.

${ }^{2}$ Org. Synth. Coll. V, 1973, 46 and 310.
} 
B. NMR Spectra for Compounds 2, 11 and $\mathbf{1 2 .}$

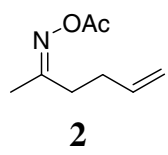

言

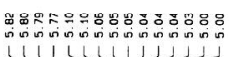

vivinum

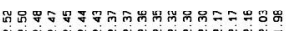

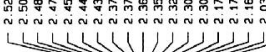

$+2$
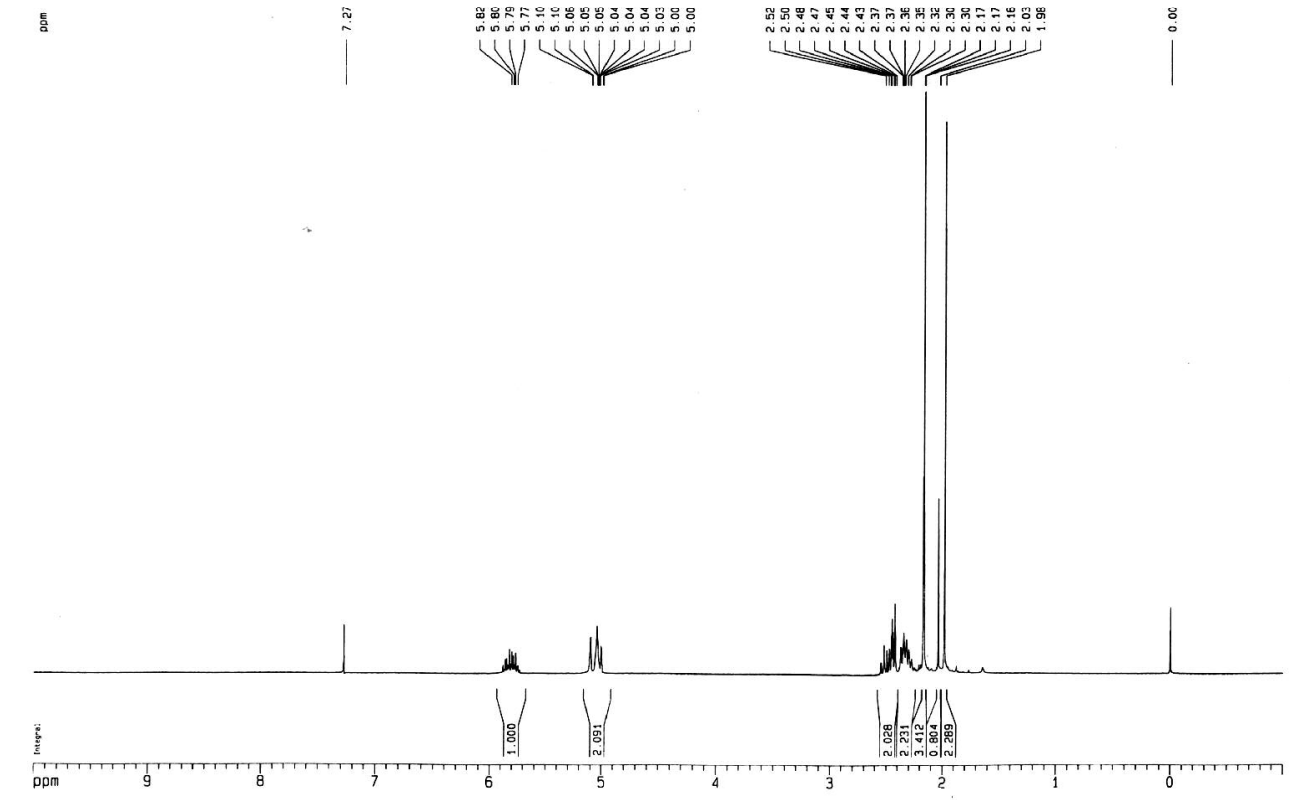

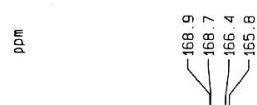

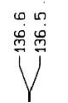

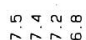

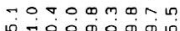

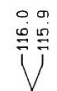

ตั

WYI

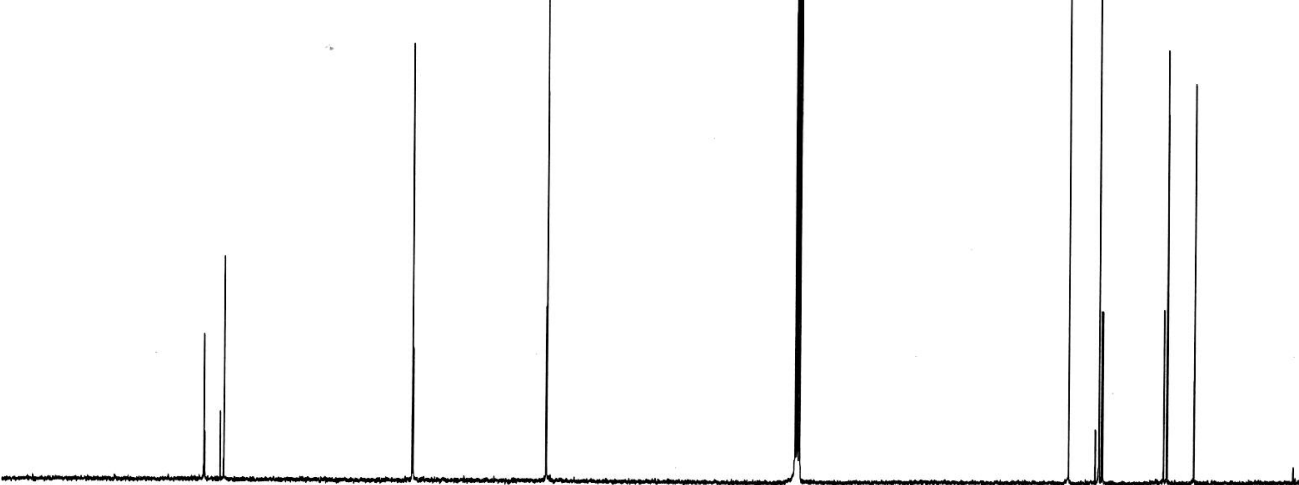

pp 180 ${ }_{160}+\frac{1}{140}$ 120 $120+{ }_{100}^{\prime}+\frac{1}{80}$ 

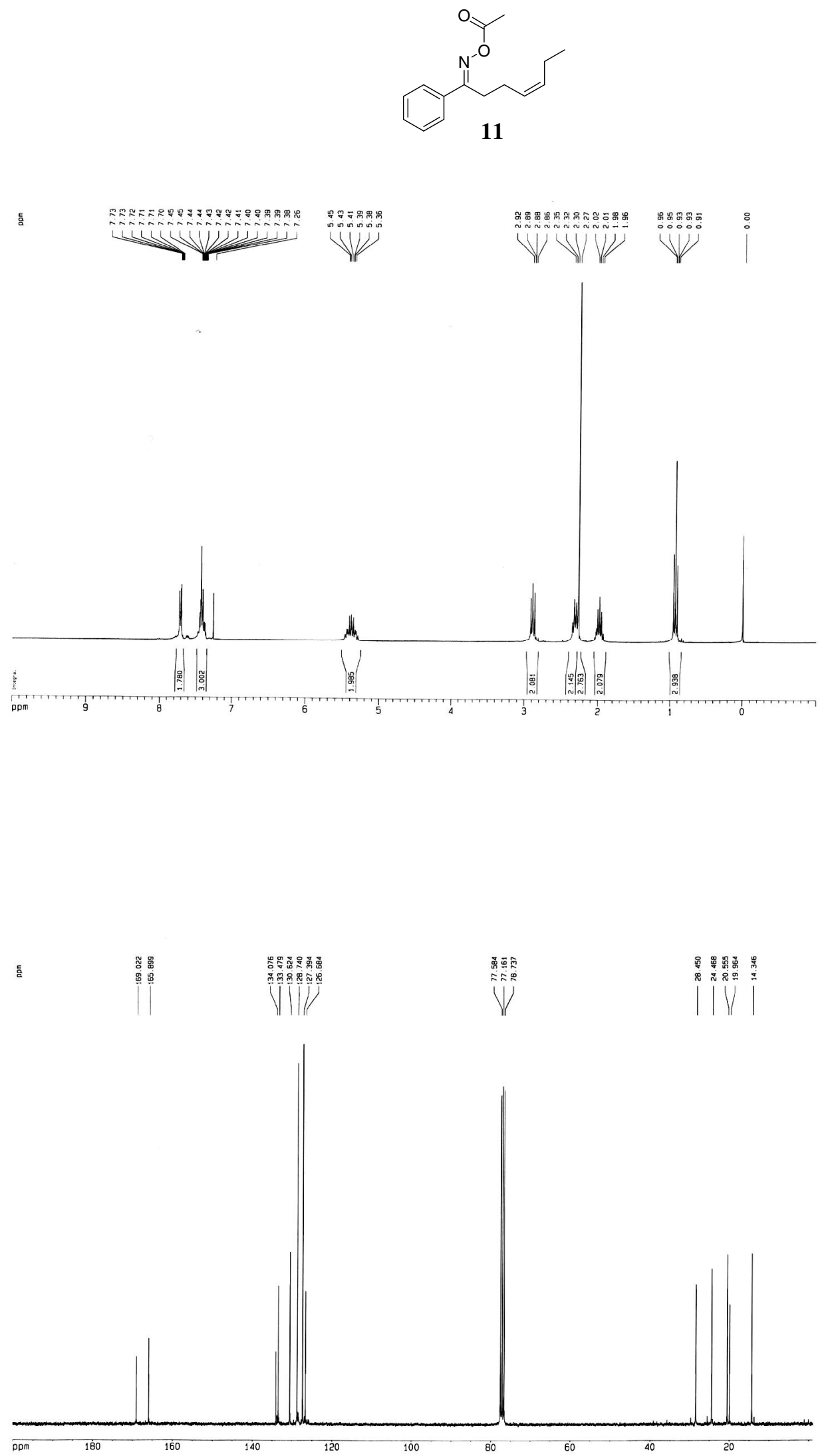


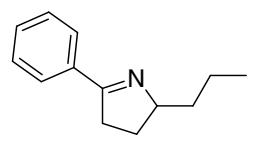

12

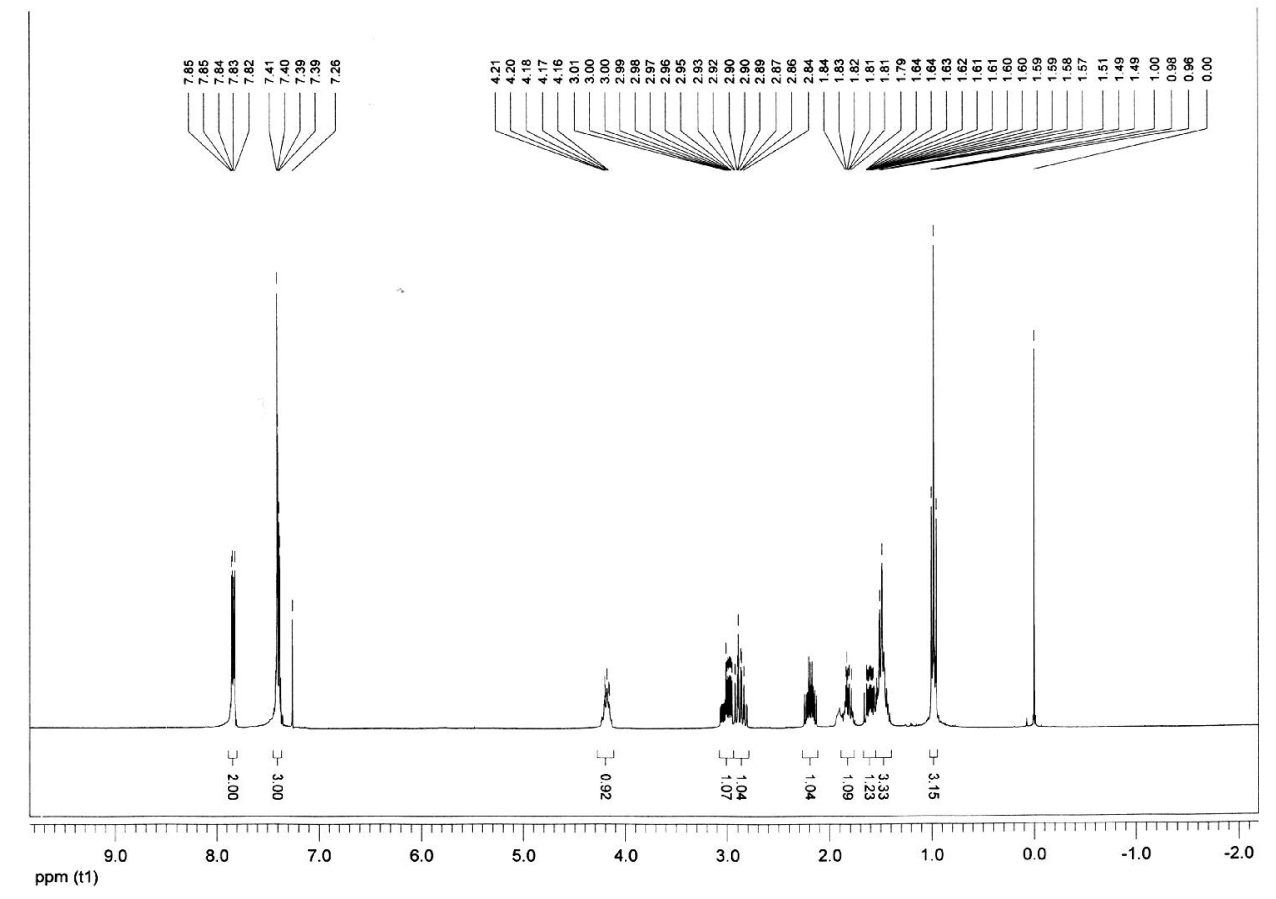

Б

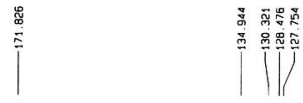

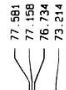

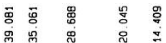

1)

||$^{\text {gi }} \mid$

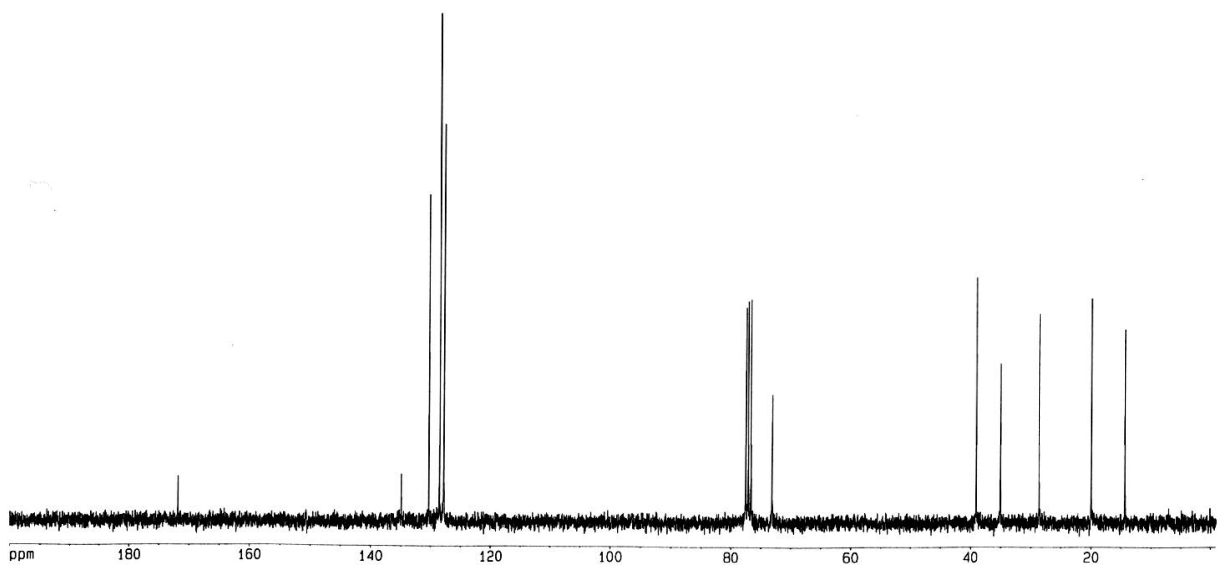


Cosy ${ }^{1} \mathrm{H}^{-1} \mathrm{H}$

QN-

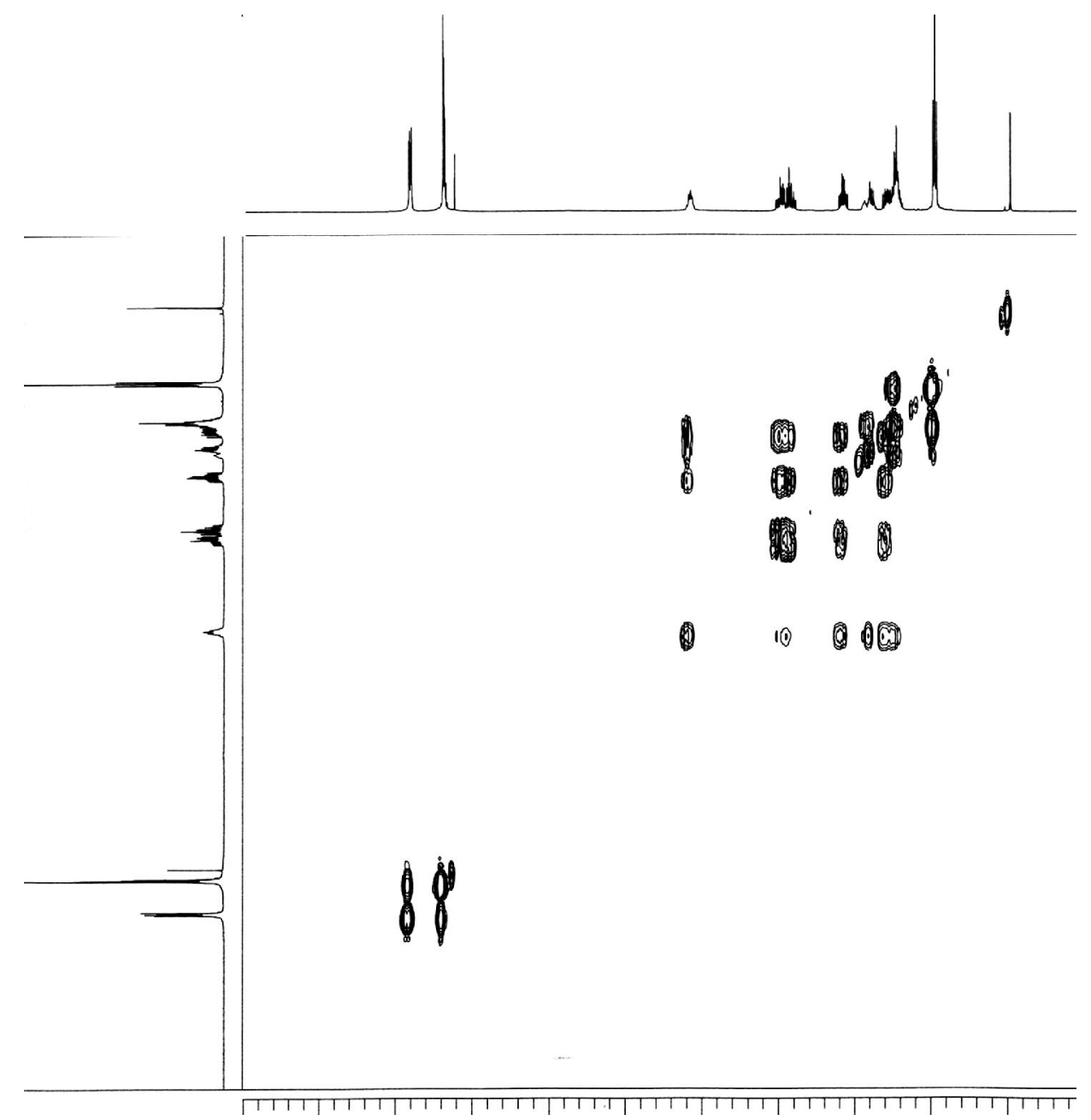


C. Experimental Procedure for Irradiation of $\mathbf{1 1}$ in the Presence of Different Triplet Quenchers and Sensitizers.

Five $1.02 \times 10^{-2} \mathrm{M}$ solutions of acyloxime $\mathbf{1 1}$ in deoxygenated acetonitrile were contained in five $20 \mathrm{ml}$ quartz tubes. About ten equivalents of 1,4-cyclohexadiene and ten equivalents of ketone (benzophenone, 2-acetylnaphthalene, 1-acetylnaphthalene and 9-fluorenone) were added to each tube. One tube was kept ketone free. The irradiation was performed for 2 hours in a merry-go-round reactor through solution filter which removes radiation wavelength under $330 \mathrm{~nm} .{ }^{3}$ The solvent was then removed from the reaction mixtures with a rotary evaporator and the amounts of $\mathbf{1 1}$ and $\mathbf{1 2}$ were quantified by ${ }^{1} \mathrm{H}$ RMN using a solution of 1,3,5-trimethoxybenzene as an internal standard.

\footnotetext{
${ }^{3}$ Concentrations: $\mathrm{NaBr} \cdot 2 \mathrm{H}_{2} \mathrm{O}, 650 \mathrm{~g} / \mathrm{L} ; \mathrm{Pb}\left(\mathrm{NO}_{3}\right)_{2}, 3 \mathrm{~g} / \mathrm{L}$. See for example: CRC Handbook of Organic Photochemistry; Scaiano, J. C.; CRC Press: Boca Raton, 1989; Vol. I, p 48
} 
D. Experimental Procedure for Laser Flash Photolysis Measurements.

The experiment was carried out using a Luzchem miniaturized Laser Flash Photolisys mLFP (LFP-111). An acetonitrile solution containing xanthone $\left(4.03 \times 10^{-4}\right.$ $\mathrm{M}$, absorbance $=0.4 \mathrm{u}$.a. at $351 \mathrm{~nm}$ ) in a quartz cuvette was purged with argon for about $10 \mathrm{~min}$. The sample was exposed to the laser pulse $(351 \mathrm{~nm}, 1 \mathrm{~Hz}, 25 \mathrm{~mJ} /$ pulse $)$ and the decay of xanthone triplet at $620 \mathrm{~nm}$ was observed. Small amounts $(1 \mu \mathrm{L})$ of the quencher 11 solution $(0.81 \mathrm{M}$ in $\mathrm{MeCN})$ were added to the solution after which the decay was measured. The quenching rate was obtained from a plot of the measured decay rates ( $\tau$ inverses) versus the quencher concentration. 
E. Experimental Procedure for Stern-Volmer Analysis.

Six $1.19 \times 10^{-2} \mathrm{M}$ solutions of acyloxime $\mathbf{1 1}$ in deoxygenated acetonitrile were placed in six $20 \mathrm{ml}$ quartz tubes. Then, 0.2, 0.4, 0.7, 2.0 and 10.0 equivalents of quencher (2,5-dimethyl-2,4-hexadiene) were added to each tube. One tube was kept quencher free. The irradiation was performed for 2 hours in a merry-go-round reactor through solution filter which removes radiation wavelength under $330 \mathrm{~nm}$. ${ }^{4}$ The solvent was then removed from the reaction mixtures with a rotary evaporator and the amounts of 11 were quantified by ${ }^{1} \mathrm{H}$ RMN using a solution of 1,3,5-trimethoxybenzene as an internal standard.

\footnotetext{
${ }^{4}$ Concentrations: $\mathrm{NaBr} \cdot 2 \mathrm{H}_{2} \mathrm{O}, 650 \mathrm{~g} / \mathrm{L} ; \mathrm{Pb}\left(\mathrm{NO}_{3}\right)_{2}, 3 \mathrm{~g} / \mathrm{L}$. See for example: CRC Handbook of Organic Photochemistry; Scaiano, J. C.; CRC Press: Boca Raton, 1989; Vol. I, p 48
} 
F. Experimental Procedure for Quantum Yield Determination.

The quantum yield of the efficiency of appearance of $\mathbf{1 2}$ was determined using a solution of trans-Azobenzene actinometer which absorbance at $358 \mathrm{~nm}$ was about 1 . The irradiation was carried out at $313 \mathrm{~nm}$ using a 500W $\mathrm{Hg}(\mathrm{Xe})$ lamp and an Oriel Cornestone 130 1/8m monochromator. trans-Azobenzene solution was irradiated for 15 minutes and the absorbance at $358 \mathrm{~nm}$ was measured (except during irradiation, only radiation $\geq 500 \mathrm{~nm}$ was admitted to the sample). Then, a solution $0.107 \mathrm{M}$ of 11 in deoxygenated acetonitrile was irradiated at $313 \mathrm{~nm}$ for 3 hours, the solvent was removed from the reaction mixture with a rotary evaporator and the amount of $\mathbf{1 2}$ were quantified by ${ }^{1} \mathrm{H}$ RMN using a solution of 1,3,5-trimethoxybenzene as an internal standard. The quantum yield value was determined by the literature method. ${ }^{5}$

\footnotetext{
${ }^{5}$ Kuhn, H.J.; Braslavsky, S.E.; Schimidt, R. Pure Appl. Chem. 2004, 76, 2105.
} 
G. Experimental Procedure for Luminescence Measurements.

Emission and excitation spectra for a $0.16 \mathrm{M}$ solution of 11 in $\mathrm{CH}_{2} \mathrm{Cl}_{2}$ were obtained on a Jobin-Yvon Horiba Fluorolog 3-11 Tau-3 spectrofluorimeter. Excitation was performed up to $320 \mathrm{~nm}$, since $\mathbf{1 1}$ does not absorb at higher wavelengths. Lifetimes were measured in phosphorimeter mode.

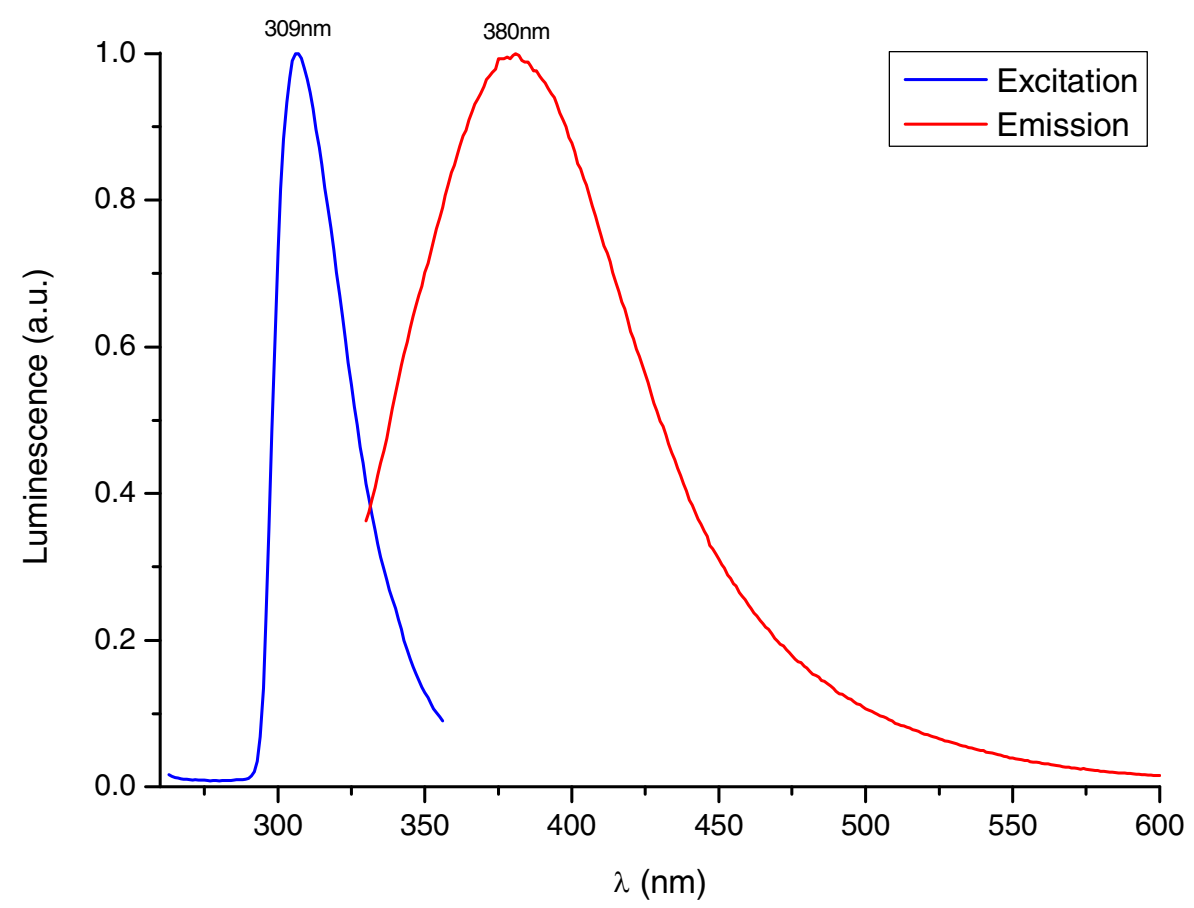

Figure 1. Emission spectra of $\mathbf{1 1}\left(0.16 \mathrm{M}\right.$ in $\left.\mathrm{CH}_{2} \mathrm{Cl}_{2}\right)$ at room temperature.

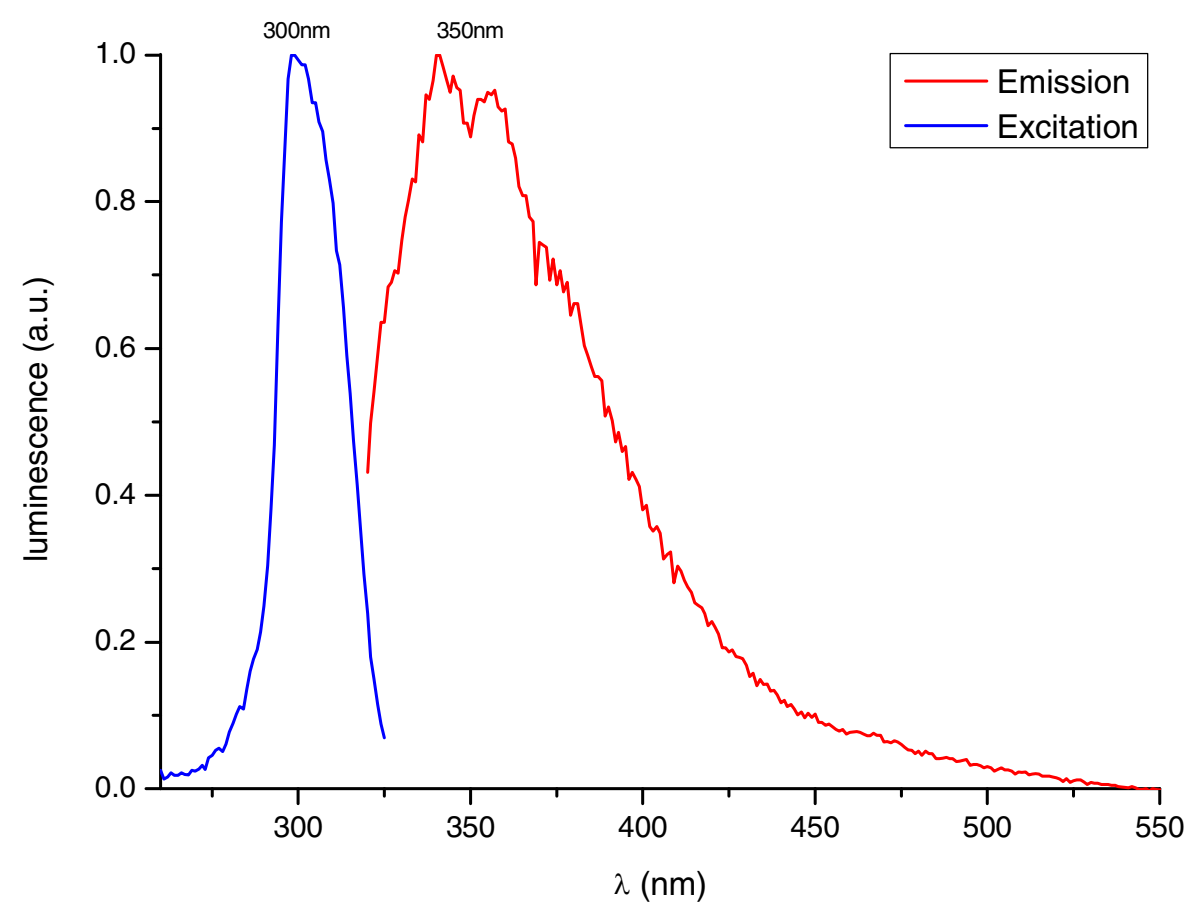

Figure 2. Emission spectra of $11\left(0.16 \mathrm{M}\right.$ in $\left.\mathrm{CH}_{2} \mathrm{Cl}_{2}\right)$ at $77 \mathrm{~K}$. 
H. Cartesian Coordinates for the Calculated Geometries.

Table 1: Cartesian coordinates for Franck-Condon geometry for model 1.

\begin{tabular}{|c|c|c|c|}
\hline Atom & $X$ & $Y$ & $\mathrm{Z}$ \\
\hline $\bar{C}$ & -1.495597 & 0.751557 & -2.820235 \\
\hline $\mathrm{C}$ & -0.528798 & -0.444478 & -2.856709 \\
\hline $\mathrm{C}$ & -2.743543 & 0.479927 & -2.023796 \\
\hline $\mathrm{C}$ & -3.158782 & 1.195941 & -0.997607 \\
\hline $\mathrm{C}$ & 0.011836 & -0.799889 & -1.501516 \\
\hline $\mathrm{N}$ & -0.129434 & -1.987153 & -1.061500 \\
\hline $\mathrm{O}$ & 0.480553 & -2.070740 & 0.237607 \\
\hline $\mathrm{C}$ & 0.375485 & -3.292094 & 0.804881 \\
\hline $\mathrm{O}$ & -0.160874 & -4.230230 & 0.308889 \\
\hline $\mathrm{C}$ & 1.041345 & -3.275248 & 2.156343 \\
\hline $\mathrm{H}$ & -1.767800 & 0.991466 & -3.845506 \\
\hline $\mathrm{H}$ & -0.988077 & 1.625404 & -2.420757 \\
\hline $\mathrm{H}$ & 0.304915 & -0.200224 & -3.510604 \\
\hline $\mathrm{H}$ & -1.018912 & -1.316328 & -3.275176 \\
\hline $\mathrm{H}$ & -3.323926 & -0.374419 & -2.336926 \\
\hline $\mathrm{H}$ & -4.063207 & 0.952468 & -0.469328 \\
\hline $\mathrm{H}$ & -2.613783 & 2.055654 & -0.645361 \\
\hline $\mathrm{H}$ & 0.516867 & -0.048979 & -0.915258 \\
\hline $\mathrm{H}$ & 0.569209 & -2.539126 & 2.796555 \\
\hline $\mathrm{H}$ & 2.086538 & -3.006801 & 2.055329 \\
\hline $\mathrm{H}$ & 0.957844 & -4.254881 & 2.603487 \\
\hline
\end{tabular}


Table 2: Cartesian coordinates for CI $\mathrm{S}_{2} / \mathrm{S}_{1}$ geometry for model 1.

\begin{tabular}{|c|c|c|c|}
\hline Atom & $\mathrm{X}$ & $\mathrm{Y}$ & $\mathrm{Z}$ \\
\hline $\mathrm{C}$ & -1.721685 & 0.307025 & -2.798928 \\
\hline $\mathrm{C}$ & -0.499708 & -0.627166 & -2.823473 \\
\hline $\mathrm{C}$ & -2.487984 & 0.224305 & -1.505140 \\
\hline $\mathrm{C}$ & -2.773664 & 1.244530 & -0.720035 \\
\hline $\mathrm{C}$ & 0.544725 & -0.227336 & -1.805600 \\
\hline $\mathrm{N}$ & 1.230793 & -1.076127 & -1.117864 \\
\hline $\mathrm{O}$ & 1.007208 & -1.690011 & 0.624354 \\
\hline $\mathrm{C}$ & 0.257209 & -2.817210 & 0.640724 \\
\hline $\mathrm{O}$ & -0.413972 & -3.193080 & -0.269444 \\
\hline $\mathrm{C}$ & 0.375581 & -3.493914 & 1.984045 \\
\hline $\mathrm{H}$ & -2.377117 & 0.023374 & -3.619504 \\
\hline $\mathrm{H}$ & -1.416761 & 1.333638 & -2.984846 \\
\hline $\mathrm{H}$ & -0.050806 & -0.600911 & -3.814039 \\
\hline $\mathrm{H}$ & -0.797175 & -1.649270 & -2.624906 \\
\hline $\mathrm{H}$ & -2.814023 & -0.764610 & -1.220357 \\
\hline $\mathrm{H}$ & -3.329877 & 1.115172 & 0.191550 \\
\hline $\mathrm{H}$ & -2.468233 & 2.249827 & -0.956697 \\
\hline $\mathrm{H}$ & 0.863155 & 0.817769 & -1.831763 \\
\hline $\mathrm{H}$ & 0.040865 & -2.825983 & 2.769745 \\
\hline $\mathrm{H}$ & 1.411314 & -3.747457 & 2.179937 \\
\hline $\mathrm{H}$ & -0.227988 & -4.390737 & 1.985053 \\
\hline
\end{tabular}


Table 3: Cartesian coordinates for CI $S_{1} / S_{0}$ geometry for model 1.

\begin{tabular}{|c|c|c|c|}
\hline Atom & $\mathrm{X}$ & $\mathrm{Y}$ & $\mathrm{Z}$ \\
\hline $\mathrm{C}$ & -1.800900 & 0.280314 & -2.852603 \\
\hline $\mathrm{C}$ & -0.461628 & -0.345425 & -3.273018 \\
\hline $\mathrm{C}$ & -2.133388 & 0.057374 & -1.402091 \\
\hline $\mathrm{C}$ & -2.426430 & 1.013644 & -0.542771 \\
\hline $\mathrm{C}$ & 0.710280 & 0.194255 & -2.482723 \\
\hline $\mathrm{N}$ & 1.531673 & -0.533591 & -1.868743 \\
\hline $\mathrm{O}$ & 0.384436 & -1.686955 & 0.999325 \\
\hline $\mathrm{C}$ & -0.116322 & -2.922839 & 1.083445 \\
\hline $\mathrm{O}$ & -0.795047 & -3.359309 & 0.178468 \\
\hline $\mathrm{C}$ & 0.182428 & -3.682489 & 2.349128 \\
\hline $\mathrm{H}$ & -2.579717 & -0.156607 & -3.474062 \\
\hline $\mathrm{H}$ & -1.792396 & 1.345536 & -3.067658 \\
\hline $\mathrm{H}$ & -0.291419 & -0.142405 & -4.327014 \\
\hline $\mathrm{H}$ & -0.492307 & -1.421893 & -3.151865 \\
\hline $\mathrm{H}$ & -2.112075 & -0.966464 & -1.065810 \\
\hline $\mathrm{H}$ & -2.656454 & 0.797326 & 0.485265 \\
\hline $\mathrm{H}$ & -2.447804 & 2.051706 & -0.829838 \\
\hline $\mathrm{H}$ & 0.863879 & 1.266220 & -2.463860 \\
\hline $\mathrm{H}$ & -0.187263 & -3.129952 & 3.204975 \\
\hline $\mathrm{H}$ & 1.253978 & -3.790352 & 2.469509 \\
\hline $\mathrm{H}$ & -0.281664 & -4.656267 & 2.314752 \\
\hline
\end{tabular}


Table 4: Cartesian coordinates for radical generated after excited state decay for model 1.

\begin{tabular}{|c|c|c|c|}
\hline Atom & $X$ & $\mathrm{Y}$ & $\mathrm{Z}$ \\
\hline $\mathrm{C}$ & -1.644832 & 0.055263 & -2.719099 \\
\hline $\mathrm{C}$ & -0.403113 & -0.849940 & -2.662685 \\
\hline $\mathrm{C}$ & -2.258148 & 0.299746 & -1.366140 \\
\hline $\mathrm{C}$ & -2.477353 & 1.490429 & -0.843652 \\
\hline $\mathrm{C}$ & 0.723748 & -0.237467 & -1.865236 \\
\hline $\mathrm{N}$ & 1.318269 & -0.816998 & -0.944211 \\
\hline $\mathrm{O}$ & -1.165942 & -1.892904 & 2.025165 \\
\hline $\mathrm{C}$ & -0.718753 & -2.796220 & 1.128616 \\
\hline $\mathrm{O}$ & -1.415693 & -3.030946 & 0.179617 \\
\hline $\mathrm{C}$ & 0.625839 & -3.381129 & 1.443310 \\
\hline $\mathrm{H}$ & -2.374962 & -0.427211 & -3.364432 \\
\hline $\mathrm{H}$ & -1.391917 & 1.002773 & -3.187460 \\
\hline $\mathrm{H}$ & -0.041573 & -1.022072 & -3.673505 \\
\hline $\mathrm{H}$ & -0.653310 & -1.813019 & -2.235448 \\
\hline $\mathrm{H}$ & -2.521256 & -0.582309 & -0.804618 \\
\hline $\mathrm{H}$ & -2.921295 & 1.604872 & 0.129037 \\
\hline $\mathrm{H}$ & -2.225584 & 2.398343 & -1.366091 \\
\hline $\mathrm{H}$ & 1.036133 & 0.770048 & -2.120391 \\
\hline $\mathrm{H}$ & 0.746009 & -3.522972 & 2.510251 \\
\hline $\mathrm{H}$ & 1.394074 & -2.706603 & 1.086309 \\
\hline $\mathrm{H}$ & 0.721520 & -4.329857 & 0.933475 \\
\hline
\end{tabular}


Table 5: Cartesian coordinates for radical model 3.

\begin{tabular}{cccc}
\hline Atom & $\mathrm{X}$ & $\mathrm{Y}$ & $\mathrm{Z}$ \\
\hline $\mathrm{C}$ & -1.454690 & 1.858552 & 0.485117 \\
$\mathrm{C}$ & -1.295956 & 0.534121 & 0.537419 \\
$\mathrm{C}$ & -0.992101 & -0.345531 & -0.640356 \\
$\mathrm{C}$ & 0.266499 & -1.210699 & -0.449640 \\
$\mathrm{C}$ & 1.529235 & -0.382449 & -0.316118 \\
$\mathrm{~N}$ & 2.368219 & -0.498441 & 0.604322 \\
$\mathrm{H}$ & -1.836366 & -1.028849 & -0.814486 \\
$\mathrm{H}$ & -0.892239 & 0.265446 & -1.547445 \\
$\mathrm{H}$ & 0.388787 & -1.867768 & -1.321945 \\
$\mathrm{H}$ & 0.169484 & -1.853329 & 0.432219 \\
$\mathrm{H}$ & -1.388013 & 0.027029 & 1.500101 \\
$\mathrm{H}$ & -1.678280 & 2.440994 & 1.375352 \\
$\mathrm{H}$ & -1.368143 & 2.409651 & -0.450366 \\
$\mathrm{H}$ & 1.709314 & 0.371942 & -1.102209 \\
\hline
\end{tabular}


Table 6: Cartesian coordinates for transition state 3-TS-B.

\begin{tabular}{cccc}
\hline Atom & $\mathrm{X}$ & $\mathrm{Y}$ & $\mathrm{Z}$ \\
\hline $\mathrm{C}$ & 1.457149 & 0.082770 & 0.059487 \\
$\mathrm{~N}$ & 1.040016 & 0.799184 & 1.011162 \\
$\mathrm{C}$ & -0.999936 & 1.055007 & 0.607113 \\
$\mathrm{C}$ & -1.357427 & -0.250831 & 0.399160 \\
$\mathrm{C}$ & -0.695959 & -1.071976 & -0.668029 \\
$\mathrm{C}$ & 0.642504 & -0.455751 & -1.111545 \\
$\mathrm{H}$ & -0.514801 & -2.081041 & -0.273323 \\
$\mathrm{H}$ & -1.341145 & -1.210712 & -1.550766 \\
$\mathrm{H}$ & 0.462771 & 0.384746 & -1.797704 \\
$\mathrm{H}$ & 1.234404 & -1.186223 & -1.673911 \\
$\mathrm{H}$ & -1.915343 & -0.778146 & 1.170683 \\
$\mathrm{H}$ & -1.359381 & 1.591941 & 1.480016 \\
$\mathrm{H}$ & -0.658343 & 1.688747 & -0.207978 \\
$\mathrm{H}$ & 2.533736 & -0.158912 & 0.057733 \\
\hline
\end{tabular}

Frequency: $490.6 i \mathrm{~cm}^{-1}$ 
Table 7: Cartesian coordinates for cyclised radical 3-Prod-B.

\begin{tabular}{cccc}
\hline Atom & $\mathrm{X}$ & $\mathrm{Y}$ & $\mathrm{Z}$ \\
\hline $\mathrm{C}$ & -0.190784 & -1.372989 & 0.001564 \\
$\mathrm{~N}$ & 1.040408 & -1.061876 & 0.095440 \\
$\mathrm{C}$ & 1.431841 & 0.340898 & 0.004983 \\
$\mathrm{C}$ & 0.344117 & 1.338469 & -0.202810 \\
$\mathrm{C}$ & -1.031699 & 0.990886 & 0.247442 \\
$\mathrm{C}$ & -1.368217 & -0.445535 & -0.175725 \\
$\mathrm{H}$ & -1.099651 & 1.051472 & 1.350453 \\
$\mathrm{H}$ & -1.773911 & 1.697739 & -0.141006 \\
$\mathrm{H}$ & -1.668981 & -0.471379 & -1.234327 \\
$\mathrm{H}$ & -2.222882 & -0.838360 & 0.390868 \\
$\mathrm{H}$ & 0.605751 & 2.359904 & -0.468658 \\
$\mathrm{H}$ & 2.001818 & 0.558181 & 0.928419 \\
$\mathrm{H}$ & 2.182857 & 0.408543 & -0.796336 \\
$\mathrm{H}$ & -0.419411 & -2.443339 & 0.049790 \\
\hline
\end{tabular}


Table 8: Cartesian coordinates for radical model 4.

\begin{tabular}{cccc}
\hline Atom & $\mathrm{X}$ & $\mathrm{Y}$ & $\mathrm{Z}$ \\
\hline $\mathrm{C}$ & 2.405675 & -0.954813 & 0.270982 \\
$\mathrm{C}$ & 1.728882 & 0.189009 & 0.398234 \\
$\mathrm{C}$ & 0.921757 & 0.838887 & -0.687483 \\
$\mathrm{C}$ & -0.509971 & 1.202214 & -0.252050 \\
$\mathrm{C}$ & -1.344557 & 0.009808 & 0.198334 \\
$\mathrm{~N}$ & -1.852361 & -0.010021 & 1.347687 \\
$\mathrm{H}$ & 1.411489 & 1.775477 & -0.993545 \\
$\mathrm{H}$ & 0.903456 & 0.196696 & -1.576988 \\
$\mathrm{H}$ & -1.031239 & 1.679713 & -1.093684 \\
$\mathrm{H}$ & -0.481332 & 1.930357 & 0.565923 \\
$\mathrm{H}$ & 1.748475 & 0.712088 & 1.356328 \\
$\mathrm{H}$ & 2.979800 & -1.371952 & 1.094406 \\
$\mathrm{H}$ & 2.415020 & -1.514469 & -0.663547 \\
$\mathrm{C}$ & -1.560755 & -1.132631 & -0.772281 \\
$\mathrm{H}$ & -2.272999 & -1.854043 & -0.365101 \\
$\mathrm{H}$ & -1.940217 & -0.751861 & -1.728177 \\
$\mathrm{H}$ & -0.612110 & -1.646694 & -0.963845 \\
\hline
\end{tabular}


Table 9: Cartesian coordinates for transition state 4-TS-B.

\begin{tabular}{cccc}
\hline Atom & $\mathrm{X}$ & $\mathrm{Y}$ & $\mathrm{Z}$ \\
\hline $\mathrm{C}$ & -1.494371 & 1.097529 & 0.587921 \\
$\mathrm{C}$ & -1.841878 & -0.209115 & 0.380322 \\
$\mathrm{C}$ & -1.160235 & -1.026542 & -0.676995 \\
$\mathrm{C}$ & 0.174930 & -0.398672 & -1.108719 \\
$\mathrm{C}$ & 1.005507 & 0.126788 & 0.066505 \\
$\mathrm{~N}$ & 0.550032 & 0.839087 & 1.009822 \\
$\mathrm{H}$ & -0.972562 & -2.032702 & -0.277169 \\
$\mathrm{H}$ & -1.794359 & -1.176189 & -1.566103 \\
$\mathrm{H}$ & -0.008203 & 0.450695 & -1.782615 \\
$\mathrm{H}$ & 0.762419 & -1.120941 & -1.687691 \\
$\mathrm{H}$ & -2.404955 & -0.738606 & 1.146641 \\
$\mathrm{H}$ & -1.862637 & 1.634877 & 1.457042 \\
$\mathrm{H}$ & -1.139258 & 1.730041 & -0.221899 \\
$\mathrm{C}$ & 2.483768 & -0.209707 & 0.046089 \\
$\mathrm{H}$ & 2.998963 & 0.250811 & 0.892351 \\
$\mathrm{H}$ & 2.624541 & -1.297675 & 0.087748 \\
$\mathrm{H}$ & 2.939493 & 0.144399 & -0.887805 \\
\hline
\end{tabular}

Frequency: $467.1 i \mathrm{~cm}^{-1}$ 
Table 10: Cartesian coordinates for cyclised radical 4-Prod-B.

\begin{tabular}{cccc}
\hline Atom & $\mathrm{X}$ & $\mathrm{Y}$ & $\mathrm{Z}$ \\
\hline $\mathrm{C}$ & 0.239160 & -0.817026 & -0.962463 \\
$\mathrm{C}$ & 0.909996 & 0.217630 & -0.078535 \\
$\mathrm{~N}$ & 0.316793 & 0.974654 & 0.761692 \\
$\mathrm{C}$ & -1.128287 & 0.900858 & 0.927727 \\
$\mathrm{C}$ & -1.860402 & -0.129896 & 0.138315 \\
$\mathrm{C}$ & -1.098596 & -1.294821 & -0.385341 \\
$\mathrm{H}$ & -0.889379 & -2.016459 & 0.427798 \\
$\mathrm{H}$ & -1.673299 & -1.846915 & -1.138496 \\
$\mathrm{H}$ & 0.080724 & -0.365611 & -1.953622 \\
$\mathrm{H}$ & 0.923987 & -1.660797 & -1.119843 \\
$\mathrm{H}$ & -2.947930 & -0.130696 & 0.150574 \\
$\mathrm{H}$ & -1.306483 & 0.765463 & 2.011602 \\
$\mathrm{H}$ & -1.524949 & 1.906541 & 0.716924 \\
$\mathrm{C}$ & 2.400414 & 0.354134 & -0.236295 \\
$\mathrm{H}$ & 2.666137 & 0.529235 & -1.287779 \\
$\mathrm{H}$ & 2.771246 & 1.182306 & 0.372200 \\
$\mathrm{H}$ & 2.908684 & -0.570917 & 0.068339 \\
\hline
\end{tabular}


Table 11: Cartesian coordinates for radical model 5.

\begin{tabular}{|c|c|c|c|}
\hline Atom & $\mathrm{X}$ & $\mathrm{Y}$ & $\bar{Z}$ \\
\hline $\mathrm{C}$ & -2.326459 & 1.031329 & 2.225905 \\
\hline $\mathrm{C}$ & -2.216265 & -0.288625 & 2.059620 \\
\hline $\mathrm{C}$ & -2.121414 & -0.984404 & 0.733147 \\
\hline $\mathrm{C}$ & -0.884459 & -1.894652 & 0.611389 \\
\hline $\mathrm{C}$ & 0.436849 & -1.136504 & 0.670438 \\
\hline $\mathrm{N}$ & 1.225549 & -1.361299 & 1.629535 \\
\hline $\mathrm{H}$ & -3.006680 & -1.622948 & 0.593007 \\
\hline $\mathrm{H}$ & -2.132526 & -0.245544 & -0.077075 \\
\hline $\mathrm{H}$ & -0.929486 & -2.457503 & -0.330188 \\
\hline $\mathrm{H}$ & -0.880787 & -2.633667 & 1.420052 \\
\hline $\mathrm{H}$ & -2.189192 & -0.933706 & 2.940342 \\
\hline $\mathrm{H}$ & -2.397648 & 1.476318 & 3.215363 \\
\hline $\mathrm{H}$ & -2.347607 & 1.714908 & 1.378500 \\
\hline $\mathrm{C}$ & 0.788212 & -0.144323 & -0.390911 \\
\hline $\mathrm{C}$ & 1.649916 & 0.919794 & -0.084663 \\
\hline $\mathrm{C}$ & 2.012064 & 1.839053 & -1.064899 \\
\hline $\mathrm{C}$ & 1.520352 & 1.709721 & -2.365651 \\
\hline $\mathrm{C}$ & 0.662150 & 0.655844 & -2.678400 \\
\hline $\mathrm{C}$ & 0.294907 & -0.264511 & -1.696856 \\
\hline $\mathrm{H}$ & 2.023099 & 1.020691 & 0.930555 \\
\hline $\mathrm{H}$ & 2.676869 & 2.661585 & -0.812486 \\
\hline $\mathrm{H}$ & 1.802567 & 2.428963 & -3.130653 \\
\hline $\mathrm{H}$ & 0.276476 & 0.547320 & -3.689236 \\
\hline $\mathrm{H}$ & -0.369049 & -1.083670 & -1.959649 \\
\hline
\end{tabular}


Table 12: Cartesian coordinates for transition state 5-TS-B.

\begin{tabular}{|c|c|c|c|}
\hline Atom & $\mathrm{X}$ & $\mathrm{Y}$ & $\mathrm{Z}$ \\
\hline $\mathrm{C}$ & -3.072818 & 1.460594 & 0.360070 \\
\hline $\mathrm{C}$ & -3.415221 & 0.145051 & 0.501989 \\
\hline $\mathrm{C}$ & -2.795295 & -0.907284 & -0.367317 \\
\hline $\mathrm{C}$ & -1.508396 & -0.405787 & -1.043903 \\
\hline $\mathrm{C}$ & -0.595628 & 0.347872 & -0.068999 \\
\hline $\mathrm{N}$ & -0.999150 & 1.280054 & 0.696023 \\
\hline $\mathrm{H}$ & -2.556556 & -1.781951 & 0.253394 \\
\hline $\mathrm{H}$ & -3.489005 & -1.274156 & -1.141262 \\
\hline $\mathrm{H}$ & -1.759984 & 0.275176 & -1.868979 \\
\hline $\mathrm{H}$ & -0.976361 & -1.252036 & -1.488579 \\
\hline $\mathrm{H}$ & -3.924051 & -0.178883 & 1.408043 \\
\hline $\mathrm{H}$ & -3.386687 & 2.196575 & 1.094719 \\
\hline $\mathrm{H}$ & -2.770340 & 1.873770 & -0.598760 \\
\hline $\mathrm{C}$ & 0.860752 & -0.004791 & -0.026922 \\
\hline $\mathrm{C}$ & 1.570445 & 0.117826 & 1.177060 \\
\hline $\mathrm{C}$ & 2.929930 & -0.177003 & 1.230580 \\
\hline $\mathrm{C}$ & 3.606464 & -0.588256 & 0.080037 \\
\hline $\mathrm{C}$ & 2.911564 & -0.705235 & -1.123534 \\
\hline $\mathrm{C}$ & 1.546727 & -0.421822 & -1.176065 \\
\hline $\mathrm{H}$ & 1.038556 & 0.442722 & 2.066722 \\
\hline $\mathrm{H}$ & 3.463653 & -0.087296 & 2.173867 \\
\hline $\mathrm{H}$ & 4.668683 & -0.816917 & 0.122051 \\
\hline $\mathrm{H}$ & 3.431757 & 1.017220 & -2.026182 \\
\hline $\mathrm{H}$ & 1.023244 & -0.507154 & -2.125167 \\
\hline
\end{tabular}

Frequency: $451.9 i \mathrm{~cm}^{-1}$ 
Table 13: Cartesian coordinates for cyclised radical 5-Prod-B.

\begin{tabular}{|c|c|c|c|}
\hline Atom & $\mathrm{X}$ & $\mathrm{Y}$ & $\mathrm{Z}$ \\
\hline $\mathrm{C}$ & -1.366905 & -1.080213 & -0.782980 \\
\hline $\mathrm{C}$ & -0.670588 & -0.024107 & 0.058333 \\
\hline $\mathrm{N}$ & -1.261116 & 0.755490 & 0.885573 \\
\hline $\mathrm{C}$ & -2.700417 & 0.713017 & 1.066723 \\
\hline $\mathrm{C}$ & -3.456120 & -0.315929 & 0.301360 \\
\hline $\mathrm{C}$ & -2.710122 & -1.507623 & -0.177763 \\
\hline $\mathrm{H}$ & -2.506488 & -2.197339 & 0.663903 \\
\hline $\mathrm{H}$ & -3.291944 & -2.083886 & -0.906618 \\
\hline $\mathrm{H}$ & -1.525759 & -0.676129 & -1.793636 \\
\hline $\mathrm{H}$ & -0.713232 & -1.952792 & -0.896402 \\
\hline $\mathrm{H}$ & -4.543187 & -0.293911 & 0.308321 \\
\hline $\mathrm{H}$ & -2.870769 & 0.599541 & 2.155096 \\
\hline $\mathrm{H}$ & -3.078342 & 1.723840 & 0.844769 \\
\hline $\mathrm{C}$ & 0.806947 & 0.131000 & -0.091197 \\
\hline $\mathrm{C}$ & 1.504366 & -0.435393 & -1.168038 \\
\hline $\mathrm{C}$ & 2.882448 & -0.258478 & -1.300907 \\
\hline $\mathrm{C}$ & 3.588739 & 0.478468 & -0.352391 \\
\hline $\mathrm{C}$ & 2.904877 & 1.045510 & 0.726826 \\
\hline $\mathrm{C}$ & 1.530233 & 0.878117 & 0.852707 \\
\hline $\mathrm{H}$ & 0.976134 & -1.008491 & -1.925065 \\
\hline $\mathrm{H}$ & 3.401969 & -0.699172 & -2.148652 \\
\hline $\mathrm{H}$ & 4.663545 & 0.611353 & -0.451412 \\
\hline $\mathrm{H}$ & 3.448169 & 1.620994 & 1.472986 \\
\hline $\mathrm{H}$ & 0.986968 & 1.321352 & 1.681664 \\
\hline
\end{tabular}


Table 14: Cartesian coordinates for radical model 6.

\begin{tabular}{cccc}
\hline Atom & $\mathrm{X}$ & $\mathrm{Y}$ & $\mathrm{Z}$ \\
\hline $\mathrm{C}$ & -2.453533 & 0.590549 & 0.339281 \\
$\mathrm{C}$ & -1.287401 & 1.335868 & 0.215620 \\
$\mathrm{C}$ & -0.040870 & 0.730636 & -0.017410 \\
$\mathrm{C}$ & 0.034635 & -0.680242 & -0.129378 \\
$\mathrm{C}$ & -1.165572 & -1.407369 & -0.046854 \\
$\mathrm{C}$ & -2.390597 & -0.795526 & 0.196467 \\
$\mathrm{C}$ & 1.262745 & -1.460742 & -0.362909 \\
$\mathrm{C}$ & 2.450289 & -1.299162 & 0.233298 \\
$\mathrm{C}$ & 1.090006 & 1.670751 & -0.212329 \\
$\mathrm{~N}$ & 2.331834 & 1.476162 & -0.256350 \\
$\mathrm{H}$ & 1.140907 & -2.296177 & -1.054036 \\
$\mathrm{H}$ & 3.280411 & -1.959392 & -0.005045 \\
$\mathrm{H}$ & 2.638560 & -0.529324 & 0.974319 \\
$\mathrm{H}$ & 0.770534 & 2.722053 & -0.337603 \\
$\mathrm{H}$ & -1.121105 & -2.488674 & -0.158879 \\
$\mathrm{H}$ & -3.292585 & -1.398166 & 0.268731 \\
$\mathrm{H}$ & -3.402479 & 1.087323 & 0.523345 \\
$\mathrm{H}$ & -1.335288 & 2.420649 & 0.288901 \\
\hline & & &
\end{tabular}


Table 15: Cartesian coordinates for transition state 6-TS-B.

\begin{tabular}{cccc}
\hline Atom & $\mathrm{X}$ & $\mathrm{Y}$ & $\mathrm{Z}$ \\
\hline $\mathrm{C}$ & 2.346185 & -0.977270 & 0.474478 \\
$\mathrm{C}$ & 1.256352 & -1.416875 & -0.220008 \\
$\mathrm{C}$ & -0.020138 & -0.728557 & -0.095566 \\
$\mathrm{C}$ & -0.024394 & 0.687014 & -0.012712 \\
$\mathrm{C}$ & 1.217462 & 1.468391 & -0.218288 \\
$\mathrm{~N}$ & 2.426782 & 1.101796 & -0.140097 \\
$\mathrm{H}$ & 1.357710 & -2.189201 & -0.981688 \\
$\mathrm{H}$ & 3.335593 & -1.380031 & 0.277756 \\
$\mathrm{H}$ & 2.230899 & -0.457395 & 1.420962 \\
$\mathrm{H}$ & 1.051474 & 2.520630 & -0.506081 \\
$\mathrm{C}$ & -1.252640 & -1.402500 & -0.119426 \\
$\mathrm{C}$ & -1.240300 & 1.372429 & 0.113890 \\
$\mathrm{C}$ & -2.451089 & -0.710297 & 0.017989 \\
$\mathrm{C}$ & -2.447397 & 0.682374 & 0.151079 \\
$\mathrm{H}$ & -1.257622 & -2.486022 & -0.216397 \\
$\mathrm{H}$ & -3.392448 & -1.254467 & 0.020126 \\
$\mathrm{H}$ & -3.383102 & 1.225694 & 0.254583 \\
$\mathrm{H}$ & -1.234231 & 2.459961 & 0.162799 \\
\hline
\end{tabular}

Frequency: $409.1 i \mathrm{~cm}^{-1}$ 
Table 16: Cartesian coordinates for cyclised radical 6-Prod-B.

\begin{tabular}{cccc}
\hline Atom & $\mathrm{X}$ & $\mathrm{Y}$ & $\mathrm{Z}$ \\
\hline $\mathrm{C}$ & -2.475202 & 0.675101 & -0.174213 \\
$\mathrm{C}$ & -1.271912 & 1.376287 & -0.082119 \\
$\mathrm{C}$ & -0.058702 & 0.695244 & 0.000821 \\
$\mathrm{C}$ & -0.030647 & -0.736996 & -0.007395 \\
$\mathrm{C}$ & -1.269996 & -1.429904 & -0.102021 \\
$\mathrm{C}$ & -2.462006 & -0.731843 & -0.183302 \\
$\mathrm{C}$ & 1.213970 & -1.368561 & 0.078064 \\
$\mathrm{C}$ & 2.480152 & -0.584526 & 0.175220 \\
$\mathrm{C}$ & 1.215385 & 1.397821 & 0.097805 \\
$\mathrm{~N}$ & 2.378189 & 0.864714 & 0.178015 \\
$\mathrm{H}$ & 1.277964 & -2.455240 & 0.074855 \\
$\mathrm{H}$ & 3.166750 & -0.857641 & -0.646298 \\
$\mathrm{H}$ & 3.041597 & -0.869886 & 1.083165 \\
$\mathrm{H}$ & 1.178850 & 2.491791 & 0.102699 \\
$\mathrm{H}$ & -1.267472 & -2.517854 & -0.109560 \\
$\mathrm{H}$ & -3.400445 & -1.276847 & -0.255093 \\
$\mathrm{H}$ & -3.417830 & 1.211809 & -0.238675 \\
$\mathrm{H}$ & -1.272987 & 2.465131 & -0.074370 \\
\hline
\end{tabular}


Table 17: Cartesian coordinates for radical model 7.

\begin{tabular}{|c|c|c|c|}
\hline Atom & $X$ & $\mathrm{Y}$ & $\mathrm{Z}$ \\
\hline $\mathrm{C}$ & -2.744146 & 0.252512 & 0.100453 \\
\hline $\mathrm{C}$ & -2.408276 & -1.089134 & -0.074378 \\
\hline $\mathrm{C}$ & -1.066240 & -1.454342 & -0.155613 \\
\hline $\mathrm{C}$ & -0.049360 & -0.496339 & -0.071320 \\
\hline $\mathrm{C}$ & -0.378379 & 0.871643 & 0.061883 \\
\hline $\mathrm{C}$ & -1.738135 & 1.211102 & 0.170341 \\
\hline $\mathrm{C}$ & 1.365770 & -1.007918 & -0.070908 \\
\hline $\mathrm{N}$ & 1.820747 & -1.585363 & -1.092579 \\
\hline $\mathrm{C}$ & 0.594506 & 1.977820 & 0.068048 \\
\hline $\mathrm{C}$ & 1.775594 & 2.031024 & -0.563507 \\
\hline $\mathrm{H}$ & 0.262785 & 2.865473 & 0.608960 \\
\hline $\mathrm{H}$ & 2.389831 & 2.925934 & -0.504455 \\
\hline $\mathrm{H}$ & 2.157341 & 1.213495 & -1.168443 \\
\hline $\mathrm{C}$ & 2.170582 & -0.912596 & 1.210806 \\
\hline $\mathrm{H}$ & -2.003767 & 2.259824 & 0.288687 \\
\hline $\mathrm{H}$ & -3.785951 & 0.553639 & 0.177552 \\
\hline $\mathrm{H}$ & -3.182577 & -1.849443 & -0.137111 \\
\hline $\mathrm{H}$ & -0.790195 & -2.499058 & -0.271854 \\
\hline $\mathrm{H}$ & 3.187858 & -1.280501 & 1.054481 \\
\hline $\mathrm{H}$ & 2.205354 & 0.123275 & 1.561311 \\
\hline $\mathrm{H}$ & 1.682592 & -1.517722 & 1.984095 \\
\hline
\end{tabular}


Table 18: Cartesian coordinates for transition state 7-TS-B.

\begin{tabular}{|c|c|c|c|}
\hline Atom & $X$ & $\mathrm{Y}$ & $\mathrm{Z}$ \\
\hline $\mathrm{C}$ & -2.315510 & -1.149160 & -0.233791 \\
\hline $\mathrm{C}$ & -0.951183 & -1.416590 & -0.282775 \\
\hline $\mathrm{C}$ & -0.000982 & -0.401662 & -0.086651 \\
\hline $\mathrm{C}$ & -0.455155 & 0.925414 & 0.136540 \\
\hline $\mathrm{C}$ & -1.835321 & 1.163088 & 0.248874 \\
\hline $\mathrm{C}$ & -2.759799 & 0.144169 & 0.054727 \\
\hline $\mathrm{C}$ & 0.518266 & 1.998615 & 0.284732 \\
\hline $\mathrm{C}$ & 1.622333 & 2.032176 & -0.511003 \\
\hline $\mathrm{C}$ & 1.449835 & -0.748862 & 0.039046 \\
\hline $\mathrm{N}$ & 2.428120 & 0.054797 & -0.074975 \\
\hline $\mathrm{H}$ & 0.414549 & 2.686027 & 1.123499 \\
\hline $\mathrm{H}$ & 2.430734 & 2.733523 & -0.323972 \\
\hline $\mathrm{H}$ & 1.618837 & 1.568398 & -1.492897 \\
\hline $\mathrm{C}$ & 1.808318 & -2.190385 & 0.373508 \\
\hline $\mathrm{H}$ & -2.175355 & 2.175393 & 0.456792 \\
\hline $\mathrm{H}$ & -3.824328 & 0.355461 & 0.121332 \\
\hline $\mathrm{H}$ & -3.030435 & -1.952088 & -0.395064 \\
\hline $\mathrm{H}$ & -0.622925 & -2.436290 & -0.461551 \\
\hline $\mathrm{H}$ & 2.880415 & -2.261298 & 0.570626 \\
\hline $\mathrm{H}$ & 1.254996 & -2.538644 & 1.252966 \\
\hline $\mathrm{H}$ & 1.571863 & -2.854874 & -0.466149 \\
\hline
\end{tabular}

Frequency: $367.6 i \mathrm{~cm}^{-1}$ 
Table 19: Cartesian coordinates for cyclised radical 7-Prod-B.

\begin{tabular}{|c|c|c|c|}
\hline Atom & $\mathrm{X}$ & $\mathrm{Y}$ & $\mathrm{Z}$ \\
\hline $\mathrm{N}$ & 2.248967 & 0.529331 & 0.166326 \\
\hline $\mathrm{C}$ & 2.348257 & -0.915662 & 0.163384 \\
\hline $\mathrm{C}$ & 1.083038 & -1.692317 & 0.066344 \\
\hline $\mathrm{C}$ & -0.153601 & -1.048295 & -0.018515 \\
\hline $\mathrm{C}$ & -0.183974 & 0.385980 & -0.010563 \\
\hline $\mathrm{C}$ & 1.100915 & 1.104311 & 0.087371 \\
\hline $\mathrm{C}$ & -1.386597 & -1.753758 & -0.112662 \\
\hline $\mathrm{C}$ & -2.587433 & -1.073904 & -0.194666 \\
\hline $\mathrm{C}$ & -2.609477 & 0.331035 & -0.186305 \\
\hline $\mathrm{C}$ & -1.412156 & 1.044188 & -0.094692 \\
\hline $\mathrm{H}$ & 1.136125 & -2.779570 & 0.062469 \\
\hline $\mathrm{H}$ & 3.034540 & -1.191160 & -0.658082 \\
\hline $\mathrm{H}$ & 2.909333 & -1.203273 & 1.071224 \\
\hline $\mathrm{C}$ & 1.095917 & 2.611339 & 0.097615 \\
\hline $\mathrm{H}$ & -1.368041 & -2.841645 & -0.119020 \\
\hline $\mathrm{H}$ & -3.519979 & -1.628951 & -0.266020 \\
\hline $\mathrm{H}$ & -3.554884 & 0.863047 & -0.250890 \\
\hline $\mathrm{H}$ & -1.444426 & 2.130017 & -0.089333 \\
\hline $\mathrm{H}$ & 2.125418 & 2.967087 & 0.174596 \\
\hline $\mathrm{H}$ & 0.648722 & 3.017017 & -0.818622 \\
\hline $\mathrm{H}$ & 0.521094 & 3.004620 & 0.945528 \\
\hline
\end{tabular}


Table 20: Cartesian coordinates for radical model $\mathbf{8}$.

\begin{tabular}{|c|c|c|c|}
\hline Atom & $\mathrm{X}$ & $\bar{Y}$ & $\bar{Z}$ \\
\hline $\mathrm{C}$ & -1.366689 & -0.858305 & -0.866719 \\
\hline $\mathrm{C}$ & -1.387380 & -0.254605 & 0.396004 \\
\hline $\mathrm{C}$ & -2.608635 & 0.182497 & 0.929511 \\
\hline $\mathrm{C}$ & -3.787569 & 0.016983 & 0.209366 \\
\hline $\mathrm{C}$ & -3.762349 & -0.588084 & -1.049824 \\
\hline $\mathrm{C}$ & -2.550714 & -1.025284 & -1.584230 \\
\hline $\mathrm{C}$ & -0.132254 & -0.114737 & 1.193704 \\
\hline $\mathrm{N}$ & -0.153459 & 0.005299 & 2.452497 \\
\hline $\mathrm{C}$ & 1.210253 & -0.211516 & 0.520903 \\
\hline $\mathrm{C}$ & 1.983319 & -1.341175 & 0.812832 \\
\hline $\mathrm{C}$ & 3.207663 & -1.556229 & 0.183725 \\
\hline $\mathrm{C}$ & 3.666250 & -0.633591 & -0.755482 \\
\hline $\mathrm{C}$ & 2.909946 & 0.500493 & -1.035829 \\
\hline $\mathrm{C}$ & 1.686674 & 0.756110 & -0.392715 \\
\hline $\mathrm{C}$ & 1.004661 & 2.021841 & -0.710735 \\
\hline $\mathrm{C}$ & 0.220043 & 2.750141 & 0.094689 \\
\hline $\mathrm{H}$ & 1.227868 & 2.416602 & -1.702998 \\
\hline $\mathrm{H}$ & -0.205558 & 3.687501 & -0.254011 \\
\hline $\mathrm{H}$ & -0.007454 & 2.459244 & 1.115657 \\
\hline $\mathrm{H}$ & 3.280011 & 1.228017 & -1.755654 \\
\hline $\mathrm{H}$ & 4.613441 & -0.792322 & -1.265172 \\
\hline $\mathrm{H}$ & 3.790271 & -2.443579 & 0.417622 \\
\hline $\mathrm{H}$ & 1.601567 & -2.062891 & 1.530043 \\
\hline $\mathrm{H}$ & -2.622610 & 0.655475 & 1.907789 \\
\hline $\mathrm{H}$ & -4.728871 & 0.363545 & 0.629056 \\
\hline $\mathrm{H}$ & -4.684385 & -0.716325 & -1.611731 \\
\hline $\mathrm{H}$ & -2.524611 & -1.498374 & -2.562907 \\
\hline $\mathrm{H}$ & -0.424773 & -1.201215 & -1.286365 \\
\hline
\end{tabular}


Table 21: Cartesian coordinates for transition state 8-TS-B.

\begin{tabular}{|c|c|c|c|}
\hline Atom & $X$ & $\bar{Y}$ & $\bar{Z}$ \\
\hline $\mathrm{C}$ & -1.822121 & -2.351233 & -0.700816 \\
\hline $\mathrm{C}$ & -0.814562 & -1.393187 & -0.727550 \\
\hline $\mathrm{C}$ & -1.034179 & -0.097288 & -0.233140 \\
\hline $\mathrm{C}$ & -2.323127 & 0.245158 & 0.254548 \\
\hline $\mathrm{C}$ & -3.303784 & -0.757818 & 0.342052 \\
\hline $\mathrm{C}$ & -3.063864 & -2.039431 & -0.137687 \\
\hline $\mathrm{C}$ & -2.585686 & 1.617301 & 0.662513 \\
\hline $\mathrm{C}$ & -2.088969 & 2.646586 & -0.078719 \\
\hline $\mathrm{C}$ & 0.108460 & 0.860545 & -0.116696 \\
\hline $\mathrm{N}$ & 0.023865 & 2.131182 & -0.061103 \\
\hline $\mathrm{H}$ & -3.071098 & 1.800610 & 1.620368 \\
\hline $\mathrm{H}$ & -2.145448 & 3.673600 & 0.271157 \\
\hline $\mathrm{H}$ & -1.878346 & 2.519705 & -1.136710 \\
\hline $\mathrm{C}$ & 1.503501 & 0.308381 & -0.005133 \\
\hline $\mathrm{H}$ & -4.278017 & -0.502544 & 0.753499 \\
\hline $\mathrm{H}$ & -3.845924 & -2.793264 & -0.087013 \\
\hline $\mathrm{H}$ & -1.632884 & -3.346687 & -1.094618 \\
\hline $\mathrm{H}$ & 0.163899 & -1.654131 & -1.121216 \\
\hline $\mathrm{C}$ & 2.526344 & 0.846516 & -0.795996 \\
\hline $\mathrm{C}$ & 3.835008 & 0.383562 & -0.666829 \\
\hline $\mathrm{C}$ & 4.137663 & -0.610936 & 0.263864 \\
\hline $\mathrm{C}$ & 3.124505 & -1.143275 & 1.063609 \\
\hline $\mathrm{C}$ & 1.813195 & -0.690928 & 0.927567 \\
\hline $\mathrm{H}$ & 2.285074 & 1.628334 & -1.510851 \\
\hline $\mathrm{H}$ & 4.619353 & 0.802520 & -1.292726 \\
\hline $\mathrm{H}$ & 5.158963 & -0.969647 & 0.367246 \\
\hline $\mathrm{H}$ & 3.355654 & -1.913047 & 1.796190 \\
\hline $\mathrm{H}$ & 1.027423 & -1.107447 & 1.552870 \\
\hline
\end{tabular}

Frequency: $358.3 i \mathrm{~cm}^{-1}$ 
Table 22: Cartesian coordinates for cyclised radical 8-Prod-B.

\begin{tabular}{|c|c|c|c|}
\hline Atom & $X$ & $\bar{Y}$ & $\bar{Z}$ \\
\hline $\mathrm{C}$ & 1.897048 & -0.781847 & 0.913579 \\
\hline $\mathrm{C}$ & 1.488472 & 0.227277 & 0.031143 \\
\hline $\mathrm{C}$ & 2.434233 & 0.795655 & -0.830943 \\
\hline $\mathrm{C}$ & 3.755139 & 0.351580 & -0.827140 \\
\hline $\mathrm{C}$ & 4.153700 & -0.655747 & 0.053169 \\
\hline $\mathrm{C}$ & 3.222707 & -1.215215 & 0.929102 \\
\hline $\mathrm{C}$ & 0.089314 & 0.755188 & 0.038500 \\
\hline $\mathrm{N}$ & -0.023008 & 2.034081 & 0.145981 \\
\hline $\mathrm{C}$ & -1.051863 & -0.174643 & -0.073955 \\
\hline $\mathrm{C}$ & -0.909265 & -1.534530 & -0.360392 \\
\hline $\mathrm{C}$ & -2.020351 & -2.377128 & -0.428446 \\
\hline $\mathrm{C}$ & -3.307732 & -1.861552 & -0.200501 \\
\hline $\mathrm{C}$ & -3.483067 & -0.515132 & 0.057871 \\
\hline $\mathrm{C}$ & -2.369166 & 0.370294 & 0.100627 \\
\hline $\mathrm{C}$ & -2.508995 & 1.749379 & 0.273513 \\
\hline $\mathrm{C}$ & -1.330921 & 2.652892 & 0.185729 \\
\hline $\mathrm{H}$ & -3.495146 & 2.186091 & 0.420968 \\
\hline $\mathrm{H}$ & -1.325868 & 3.369872 & 1.024435 \\
\hline $\mathrm{H}$ & -1.409496 & 3.303791 & -0.707225 \\
\hline $\mathrm{H}$ & -4.480426 & -0.108190 & 0.211584 \\
\hline $\mathrm{H}$ & -4.170771 & -2.522271 & -0.240568 \\
\hline $\mathrm{H}$ & -1.886335 & -3.431816 & -0.654105 \\
\hline $\mathrm{H}$ & 0.080367 & -1.944027 & -0.539493 \\
\hline $\mathrm{H}$ & 2.122070 & 1.593990 & -1.498819 \\
\hline $\mathrm{H}$ & 4.476188 & 0.796796 & -1.508928 \\
\hline $\mathrm{H}$ & 5.185583 & -0.999147 & 0.060544 \\
\hline $\mathrm{H}$ & 3.528625 & -1.988791 & 1.629799 \\
\hline $\mathrm{H}$ & 1.180741 & -1.213687 & 1.608814 \\
\hline
\end{tabular}


Table 23: Cartesian coordinates for transition state 3-TS-A.

\begin{tabular}{cccc}
\hline Atom & $\mathrm{X}$ & $\mathrm{Y}$ & $\mathrm{Z}$ \\
\hline $\mathrm{C}$ & -1.912506 & -0.207442 & 0.556283 \\
$\mathrm{C}$ & -1.029588 & 0.017733 & -0.479394 \\
$\mathrm{C}$ & -0.045307 & 1.172019 & -0.443290 \\
$\mathrm{C}$ & 1.173244 & 0.780093 & 0.402411 \\
$\mathrm{C}$ & 1.375988 & -0.696598 & 0.156611 \\
$\mathrm{~N}$ & 0.462364 & -1.422814 & -0.316653 \\
$\mathrm{H}$ & 0.286400 & 1.396810 & -1.463034 \\
$\mathrm{H}$ & -0.519308 & 2.078162 & -0.048005 \\
$\mathrm{H}$ & 0.996438 & 0.933928 & 1.477363 \\
$\mathrm{H}$ & 2.068400 & 1.360543 & 0.145828 \\
$\mathrm{H}$ & -1.299585 & -0.370203 & -1.458798 \\
$\mathrm{H}$ & -2.682503 & -0.969720 & 0.482963 \\
$\mathrm{H}$ & -1.801929 & 0.291418 & 1.517324 \\
$\mathrm{H}$ & 2.344553 & -1.156076 & 0.407201 \\
& & & \\
\hline
\end{tabular}

Frequency: $502.7 i \mathrm{~cm}^{-1}$ 
Table 24: Cartesian coordinates for cyclised radical 3-Prod-A.

\begin{tabular}{cccc}
\hline Atom & $\mathrm{X}$ & $\mathrm{Y}$ & $\mathrm{Z}$ \\
\hline $\mathrm{C}$ & -1.929113 & 0.069160 & 0.509446 \\
$\mathrm{C}$ & -0.812242 & 0.033251 & -0.479970 \\
$\mathrm{C}$ & 0.214752 & 1.187635 & -0.329358 \\
$\mathrm{C}$ & 1.427614 & 0.521493 & 0.344482 \\
$\mathrm{C}$ & 1.115331 & -0.940027 & 0.142003 \\
$\mathrm{~N}$ & -0.042942 & -1.232017 & -0.300977 \\
$\mathrm{H}$ & 0.492965 & 1.573579 & -1.315930 \\
$\mathrm{H}$ & -0.188438 & 2.026030 & 0.246973 \\
$\mathrm{H}$ & 1.502991 & 0.745107 & 1.418528 \\
$\mathrm{H}$ & 2.388398 & 0.805213 & -0.101977 \\
$\mathrm{H}$ & -1.236127 & 0.000890 & -1.492747 \\
$\mathrm{H}$ & -2.780382 & -0.594719 & 0.392735 \\
$\mathrm{H}$ & -1.803840 & 0.573590 & 1.464412 \\
$\mathrm{H}$ & 1.826973 & -1.734641 & 0.375232 \\
\hline & & & \\
\hline
\end{tabular}


Table 25: Cartesian coordinates for transition state 6-TS-A.

\begin{tabular}{|c|c|c|c|}
\hline Atom & $\mathrm{X}$ & $\mathrm{Y}$ & $\bar{Z}$ \\
\hline $\mathrm{C}$ & -0.817043 & -1.458473 & -0.200889 \\
\hline $\mathrm{C}$ & -2.162689 & -1.115882 & -0.037854 \\
\hline $\mathrm{C}$ & -2.544405 & 0.217476 & 0.133971 \\
\hline $\mathrm{C}$ & -1.580278 & 1.225262 & 0.151452 \\
\hline $\mathrm{C}$ & -0.238158 & 0.877641 & -0.004730 \\
\hline $\mathrm{C}$ & 0.154371 & -0.458321 & -0.189498 \\
\hline $\mathrm{C}$ & 0.920968 & 1.776912 & 0.014949 \\
\hline $\mathrm{N}$ & 2.097612 & 1.313480 & -0.091453 \\
\hline $\mathrm{C}$ & 1.613728 & -0.668412 & -0.366391 \\
\hline $\mathrm{C}$ & 2.342660 & -1.459990 & 0.498041 \\
\hline $\mathrm{H}$ & 1.991826 & -0.549202 & -1.383594 \\
\hline $\mathrm{H}$ & 3.374498 & -1.719485 & 0.281002 \\
\hline $\mathrm{H}$ & 1.944958 & -1.746451 & 1.468160 \\
\hline $\mathrm{H}$ & 0.779864 & 2.862442 & 0.118081 \\
\hline $\mathrm{H}$ & -0.526849 & -2.496659 & -0.342104 \\
\hline $\mathrm{H}$ & -2.919983 & -1.896128 & -0.047245 \\
\hline $\mathrm{H}$ & -3.594651 & 0.469023 & 0.258619 \\
\hline $\mathrm{H}$ & -1.867873 & 2.264816 & 0.292935 \\
\hline
\end{tabular}

Frequency: $520.6 i \mathrm{~cm}^{-1}$ 
Table 26: Cartesian coordinates for cyclised radical 6-Prod-A.

\begin{tabular}{|c|c|c|c|}
\hline Atom & $\mathrm{X}$ & $\mathrm{Y}$ & $\bar{Z}$ \\
\hline $\mathrm{C}$ & 0.151830 & -0.428066 & -0.193684 \\
\hline $\mathrm{C}$ & -0.746350 & -1.487791 & -0.223059 \\
\hline $\mathrm{C}$ & -2.107801 & -1.207110 & -0.063623 \\
\hline $\mathrm{C}$ & -2.558774 & 0.106161 & 0.120452 \\
\hline $\mathrm{C}$ & -1.657263 & 1.171292 & 0.152409 \\
\hline $\mathrm{C}$ & -0.301283 & 0.885366 & -0.005595 \\
\hline $\mathrm{C}$ & 0.902420 & 1.720486 & -0.020271 \\
\hline $\mathrm{N}$ & 2.001524 & 1.074155 & -0.195698 \\
\hline $\mathrm{C}$ & 1.653503 & -0.366886 & -0.334524 \\
\hline $\mathrm{C}$ & 2.416809 & -1.198855 & 0.641430 \\
\hline $\mathrm{H}$ & 1.954841 & -0.643687 & -1.357760 \\
\hline $\mathrm{H}$ & 3.470555 & -1.395051 & 0.471369 \\
\hline $\mathrm{H}$ & 2.001256 & -1.405157 & 1.622872 \\
\hline $\mathrm{H}$ & 0.909205 & 2.803779 & 0.100724 \\
\hline $\mathrm{H}$ & -0.407041 & -2.511652 & -0.363815 \\
\hline $\mathrm{H}$ & -2.828791 & -2.021057 & -0.082536 \\
\hline $\mathrm{H}$ & -3.622721 & 0.294968 & 0.241019 \\
\hline $\mathrm{H}$ & -2.006510 & 2.191191 & 0.296811 \\
\hline
\end{tabular}

